\title{
Registration and Fusion of SPECT, High-Resolution MRI, and Interventional MRI for Thermal Ablation of Prostate Cancer
}

\author{
Baowei Fei, Zhenghong Lee, Daniel T. Boll, Jeffery L. Duerk, D. Bruce Sodee, Jonathan S. Lewin, and \\ David L. Wilson
}

\begin{abstract}
We are investigating interventional MRI (iMRI) guided radiofrequency thermal ablation for the minimally invasive treatment of the prostate cancer. Nuclear medicine can detect and localize tumor in the prostate not reliably seen in MRI. We intend to combine the advantages of functional images such as nuclear medicine SPECT with iMRI-guided treatments. Our concept is to first register the low-resolution SPECT with a high-resolution MRI volume. Then by registering the high-resolution MR image with live-time iMRI acquisitions, we can, in turn, map the functional data and high-resolution anatomic information to live-time iMRI images for improved tumor targeting. For the first step, we used a three-dimensional mutual information registration method. For the latter, we developed a robust slice to volume (SV) registration algorithm with special features. The concept was tested using image data from three patients and three volunteers. The SV registration accuracy was $0.4 \mathrm{~mm} \pm 0.2 \mathrm{~mm}$ as compared to our volume-to-volume registration that was previously shown to be quite accurate for these image pairs. With our image registration and fusion software, simulation experiments show that it is quite feasible to incorporate SPECT and high-resolution MRI into the iMRI-guided minimally invasive treatment procedures.
\end{abstract}

Index Terms-Fusion visualization, image-guided minimally invasive therapy, image registration, interventional MRI, prostate cancer, SPECT.

\section{INTRODUCTION}

W E USE an interventional magnetic resonance imaging (iMRI) system to guide minimally invasive treatments, including the radiofrequency (RF) thermal ablation of abdominal cancers [1]-[3]. The iMRI system consists of a $0.2 \mathrm{~T}$, clinical C-arm open MRI scanner, an in-room RF-shielded liquid crystal monitor, an MR compatible mouse, a foot pedal, and

Manuscript received June 27, 2003; revised July 28, 2003. This work was supported in part by the DOD Grant DAMD17-02-1-0230 to B. W. Fei, the NIH Grants R01-CA84433 to D. L. Wilson and R33-CA88144 to J. L. Duerk, and the ASC Grant IRG91-022-06 to Z. Lee.

B. W. Fei is with Department of Biomedical Engineering, Case Western Reserve University, Cleveland, OH 44106 USA (e-mail: Baowei.Fei@ case.edu).

Z. Lee, J. L. Duerk, and D. L. Wilson are with the Departments of Biomedical Engineering and Radiology, Case Western Reserve University and University Hospitals of Cleveland, Cleveland, OH 44106 USA (e-mail: lee@uhrad.com; duerk@uhrad.com; dlw@po.cwru.edu).

D. T. Boll and D. B. Sodee are with the Department of Radiology, Case Western University and University Hospitals of Cleveland, Cleveland, $\mathrm{OH}$ 44106 USA (e-mail: boll@uhrad.com; sodee@uhrad.com).

J. S. Lewin is with the Departments of Radiology, Biomedical Engineering, and Oncology and Neurological Surgery, Case Western Reserve University and University Hospitals of Cleveland, Cleveland, OH 44106 USA (e-mail: lewin@uhrad.com).

Digital Object Identifier 10.1109/TNS.2003.823027 an RF ablation device. We are currently investigating the extension of these techniques to the treatment of prostate cancer. Since MRI does not reliably show prostate tumors, we intend to incorporate nuclear medicine SPECT or MR spectroscopy images with higher sensitivity for detecting and localizing prostate tumors [4], [5].

To incorporate image data from other sources in a live-time iMRI procedure, we intend to register two-dimensional (2-D) slice images quickly acquired on the iMRI scanner in live-time with a previously acquired volume of image data. Then, to incorporate an image volume from another modality, it can be registered with the full MR volume. Thus, to incorporate SPECT in an iMRI procedure, we will first register the SPECT image volume with a high-resolution MR volume; then, when we register iMRI slice images to the high-resolution MR volume, we can also map them to the SPECT functional image data. If this procedure is successful, then a variety of potential visualization tools can help the physician appropriately localize and apply treatments. The live-time iMRI images will be used for guidance, and very probably any small misregistration errors can be mentally corrected by the physician. To simplify and possibly improve the slice-to-volume (SV) registration step, we intend to always use MR images acquired with similar pulse sequences.

The application of SV registration methods to iMRI-guided treatment of prostate cancer raises several challenges. First, iMRI images often have lower signal to noise ratio (SNR) than diagnostic MR images because of the emphasis on fast imaging and because of the typically lower field strength of open iMRI magnets. Second, a single slice, or a few slices, provides many fewer structures than an entire volume for voxel based matching. Third, the prostate can move relative to the pelvic bones due to changes in rectal and bladder filling [6] or changes in patient posture for treatment [7]. That is, alignment of the pelvic bones, prominent anatomical features in MR grayscale images, does not necessarily ensure that the prostate is aligned. Fourth, the normal prostate is a small organ; when healthy, it measures only $\approx 3.8 \mathrm{~cm}$ in its widest dimension [8]. The small prostate is located below the much larger bladder that can change its shape and size during imaging. Finally, times for registration and algorithm robustness are of particular concern for this application.

Previous success with registering one MR prostate volume to another [7] encourages us to pursue this plan. We call this volume-to-volume registration, or VV. We used a rigid body, mutual information registration method with some features to 
improve robustness [7]. We carefully evaluated registration quality using a variety of methods. For volume pairs acquired over a short time span from a supine subject with legs flat on the table, registration accuracy of both prostate centroids (typically $<1 \mathrm{~mm}$ ) and bony landmarks (average $1.6 \mathrm{~mm}$ ) was on the order of a voxel $(\approx 1.4 \mathrm{~mm})$. For volumes acquired under very different conditions, e.g., legs flat and legs raised into the treatment position, or with and without bladder or rectal filling, we obtained somewhat larger prostate centroids registration errors of about $3.0 \mathrm{~mm}$. From our results with VV prostate registration, we decided that we could assess SV accuracy by comparing results to $\mathrm{VV}$ registration for those volume pairs having low VV registration error.

In the next sections, we will report algorithms and results for the slice-to-volume registration between an iMRI thick slice and a high-resolution MRI volume, the three-dimensional (3-D) registration of SPECT and high-resolution MRI volumes, and the fusion of the three modalities for potential applications in iMRI-guided thermal ablation of the prostate.

\section{REGISTRATION ALGORITHMS}

\section{A. Similarity Measures}

For slice to volume registration of iMRI image slice and high-resolution MRI volume, we used two similarity measures, mutual information and correlation coefficient, in our registration. Suppose one image $R$ is the reference, and the other $F$ is floating. Their mutual information $\operatorname{MI}(R, F)$ is [9]

$$
\mathrm{MI}(R, F)=\sum_{r, f} p_{R F}(r, f) \log \frac{p_{R F}(r, f)}{p_{R}(r) \cdot p_{F}(f)} .
$$

The joint probability $p_{R F}(r, f)$ and the marginal probabilities $p_{R}(r)$ of the reference image and $p_{F}(f)$ of the floating image, can be estimated from the normalized joint intensity histogram.

The correlation coefficient (CC) is the measure of the interdependence of two random variables that ranges in value from -1 to +1 , indicating perfect negative correlation at -1 , absence of correlation at zero, and perfect positive correlation at +1 . For the reference and floating images, $R$ and $F$, their correlation coefficient $\mathrm{CC}(R, F)$ is [10]

$$
\mathrm{CC}(R, F)=\frac{\sum_{i=0}^{N-1}(R(i)-\bar{R})(F(i)-\bar{F})}{\sqrt{\left[\sum_{i=0}^{N-1}(R(i)-\bar{R})^{2}\right]\left[\sum_{i=0}^{N-1}(F(i)-\bar{F})^{2}\right]}} .
$$

Here $\bar{R}, \bar{F}$ denote the average intensities of the reference and floating volumes and the summation includes all $N$ voxels within the overlap of both volumes.

\section{B. Registration of iMRI Slice and High-Resolution MRI Volume}

We used a registration algorithm similar to the one as previously reported by us [11], [12]. We used multiresolution approach and perform registration from low to high resolution. At $1 / 4$ resolution, we resampled images so as to give $1 / 4$ number of the voxels along each linear dimension. At full resolution, we used the full number of voxels. We use correlation coefficient at the two lower resolutions because it gives fewer local maximums and because it can be calculated faster than MI. We use MI at full resolution because the peaked similarity function gives a more precise solution than CC [7]. To avoid local maximums, we include a restarting feature where registration is restarted with randomly perturbed parameters obtained from a uniform distribution about the initial transformation values at the current resolution being used. The algorithm restarts until the absolute $\mathrm{CC}$ is above an experimentally determined threshold or the maximum number of restarts is reached. Absolute CC is used rather than MI because it has a well-defined range between 0 and 1 and because it provides an independent check of the MI result at the highest resolution.

We record all important results following an optimization cycle including the CC and/or MI values, the number of restarts, and the transformation parameters. At the end of processing at a lower resolution, we always select the transformation parameters having the maximum $\mathrm{CC}$ value. We then scale the translation parameters appropriately and assign the new parameters to be initial values at the next higher resolution. At the highest resolution, $\mathrm{MI}$ instead of $\mathrm{CC}$ is the similarity measure, and we select the final transformation parameters to be those with the maximum MI value.

Typical parameter values are now described. We use an initial guess assuming an identity transformation, i.e., all initial translation and rotation parameters are zero, because the patient is normally oriented approximately the same way from one scan to the next. The algorithm restarts until the absolute $\mathrm{CC}$ is above a threshold of 0.5 or maximum numbers of restarts are reached $(10,5$, and 3 , from low to high resolution, respectively).

\section{Registration of SPECT and High-Resolution MRI Volume}

The mutual information algorithm was used to register MRI and SPECT volume images because of its ability to align multimodality images [13], [14]. Registration of SPECT and MR images is challenging because the two image types have different spatial resolutions and image features. The radiotracer used for SPECT imaging was ProstaScint ${ }^{\circledR}$ (Cytogen Corporation, Princeton, NJ), a monoclonal antibody that binds to prostate-specific membrane antigen (PSMA). Before registration, both SPECT and MRI volumes were resized using trilinear interpolation to create volumes matrix of $128 \times 128 \times 128$ with $3 \mathrm{~mm}$ isotropic voxels, a voxel size between that of the two scans. The standard parameter set for automatic registration included: 256 intensity levels for each volume, the entire 2-D joint histogram, the full field of view of $128 \times 128 \times 128$ voxels for both volumes, and no masking or cropping of either volume. Phantom data were preprocessed in a similar fashion.

\section{EXPERIMENTAL METHODS}

\section{A. High-Resolution MR Image Acquisitions}

High-resolution MRI volumes were acquired using a $1.5 \mathrm{~T}$ Siemens MRI system (Magnetom Symphony, Siemens Medical Systems, Erlangen, Germany). An 8-element phased array body coil was used to ensure coverage of the prostate with a uniform sensitivity. Typically two anterior and two posterior elements were enabled for signal acquisition. We used two different MR sequences. 
First, we used a 3-D rapid gradient echo sequence (PSIF) designed to acquire the spin-echo component of the steady state response, rather than the free induction decay. The spin echo component forms immediately prior to the RF pulse; it is shifted toward the prior RF pulse through appropriate gradient waveform design. The sequence with 9.4/5.0/60 (TR/TE/flip) yields $160 \times 256 \times 128$ voxels over a $219 \times 350 \times 192-\mathrm{mm}$ rectangular FOV and $1.4 \times 1.4 \times 1.5-\mathrm{mm}$ voxels oriented to give the highest resolution for transverse slices. There is over sampling at $31 \%$ in the slice direction to reduce aliasing artifacts. The acquisition time is $4 \mathrm{~min}$ and $15 \mathrm{sec}$. This sequence gave excellent image contrast for the prostate and its surroundings. It was used to acquire volumes for volunteers S1-S3.

Second, we used a 3-D RF spoiled gradient echo steady state pulse sequence (FLASH) with TR/TE/flip parameters of $12 / 5.0 / 60$ which give $256 \times 256 \times 128$ voxels over a $330 \times 330 \times 256-\mathrm{mm}$ field of view $(\mathrm{FOV})$ to yield $1.3 \times 1.3 \times 2.0-\mathrm{mm}$ voxels oriented to give the highest resolution for transverse slices. The acquisition time is $5 \mathrm{~min}$ and 38 sec. This sequence is good for pelvic imaging but is not ideal for the prostate. It was used to acquire volumes for patients S4-S8.

When acquiring high-resolution MR volumes, volunteers laid supine in a manner similar to the diagnostic position in routine MR scanning. Between volume acquisitions, volunteers got up from the MR table, stretched, and walked around to ensure that they would assume a different position when they laid back on the table. The coil array was centered on the prostate. We acquired three volumes from each of the volunteers S1-S3. For patients $\mathrm{S} 4-\mathrm{S} 8$, we acquired nine MRI volumes and each patient with at least one volume.

\section{B. Interventional MRI Image Acquisitions and Simulation}

We acquired iMRI images using a clinical 0.2 T C-arm open MR scanner (Siemens Open Symphony, Erlangen, Germany) modified for interventional MRI procedures and in this paper referred to as the iMRI system. We used a two-dimensional PSIF with 15.2/7.4/45 (TR/TE/FA) for image slice acquisitions. The iMRI slices were $128 \times 128$ with in-plane pixel size of $2.8 \times 2.8 \mathrm{~mm}$ and with effective slice thickness of $5 \mathrm{~mm}$.

We acquired iMRI images under the conditions simulating the treatment application. The volunteer was supine, and his legs were supported at $30^{\circ}-60^{\circ}$ relative to the horizon and separated in a "V" with an angle of $60^{\circ}-90^{\circ}$ between two legs. This is similar to the lithotomy position used in prostate therapies, and it should provide access for needle insertion in brachytherapy or RF thermal ablation. We call this the treatment position. For each of the volunteers S1-S3, we acquired 30 iMRI image slices covering the prostate. They included 10 transverse, 10 coronal, and 10 sagittal image slices. We call these images "actual" iMRI images to differentiate them from "simulated" images as described in the next paragraph.

To test a variety of clinical conditions, we used high-resolution MRI volumes to simulate iMRI images by creating thick slices and adding noise and receive coil inhomogeneity [15]. Clinically, we typically use an iMRI slice thickness of $4.0-6.0 \mathrm{~mm}$. We averaged three slices $1.4 \mathrm{~mm}$ thick to create a $4.2 \mathrm{~mm}$ thick slice. We added noise to the simulated iMRI image.

\section{SPECT Image Acquisition}

The study included five patients (S4-S8) with either high Gleason scores $(>5)$ from biopsy or rising PSA level (>10 mcg/L, prostate specific antigen) or palpation staging beyond stage T1. After patient eligibility was established, patients gave informed consent. The Institutional Review Board of the University Hospitals of Cleveland approved the imaging protocol.

Approximately four days after injecting $5 \mathrm{mCi}$ ProstaScint ${ }^{\circledR}$, the abdominal and pelvic regions were scanned using a two-head Siemens E.CAM ${ }^{+}$camera (Siemens Medical System, Inc., Hoffman Estates, IL). ProstaScint ${ }^{\circledR}$ is an [In-111]-labeled monoclonal antibody capromab penditide ( ${ }^{111}$ In MoAb 7E11.C5) used for imaging prostate cancer. The evening before scanning, patients performed a bowel prep with Fleet R Prep Kit \#3 (Fleet Pharmaceuticals, Lynchburg, VA). Images were acquired with a medium energy collimator and $15 \%$ energy window. The acquisition parameters included a step-and-shoot motion, a $128 \times 128$ pixel matrix for each projection, an imaging time of $25 \mathrm{sec}$ per stop, and a total of 120 stops over a full $360^{\circ}$ rotation. The field of view of was $53.3 \times 38.7 \mathrm{~cm}^{2}$. The ordered subsets expectation maximization (OSEM) algorithm was used for image reconstruction [16]. SPECT images were comprised of $4.795 \times 4.795 \times 4.795-\mathrm{mm}$ isotropic voxels. Each patient had one SPECT scan of the pelvis.

To analyze and validate registration of high-resolution MRI and SPECT under a controlled situation, an acrylic phantom of the pelvis and lower abdomen was used. Spheres of proportional size representing portions of the bladder, acetabula, rectum, and the prostate gland were placed in appropriate positions in the torso phantom. The spheres of acetabulum were filled with potassium phosphate. Other spheres were filled with water. The torso phantom was filled with a small amount of copper sulfate dissolved in deionized water. The SPECT scan was conducted after injecting all spheres with [In-111]-DTPA at relative concentrations comparable to those detected in human scans. The water in the torso was given a background activity of $1 \mu \mathrm{Ci} / \mathrm{ml}$ such as to mimic the background in human SPECT scans.

\section{Registration Experiments}

We used nine pairs of high-resolution MR volumes of volunteers S1-S3 to perform SV registration experiments. For each volume pair, we extracted data from one volume to simulate thick iMRI image slices; and then we registered the simulated image slices to the other volume. We desire an iMRI slice image acquisition method that gives robust, accurate registrations and is relatively insensitive to acquisition parameters. Hence, we performed experiments to determine the dependence on slice orientation (transverse, sagittal, and coronal), on slice position relative to the prostate (above, centered, and below), and on image noise from fast imaging techniques.

We also performed SV registration experiments using the actual iMRI images from volunteers S1-S3. We registered actual 
iMRI image slices with high-resolution (1.5 T system) MR volumes of the same volunteer and visually evaluated results. For each volunteer, there were three high-resolution MR volumes and 30 iMRI image slices giving $90 \mathrm{SV}$ registration experiments, and a total of 270 experiments.

A number of technical issues were examined for MI registration of MRI and ProstaScint ${ }^{\circledR}$ SPECT prostate images. First, MRI acquisition, by varying the MR imaging pulse sequence, various structures can be emphasized or suppressed. Several different acquisition sequences were tested and its effect on registration accuracy and robustness was determined. Second, because of the different dynamic ranges between MR and SPECT images, intensity scaling was studied for its effect on registration. This is prompted by a recent study showing that scaling images to 16 gray levels gives better results than 256 gray levels when registering muscle fiber images [17]. Third, because of the sparseness in the histogram, the use of a portion or a section rather than the full joint histogram was evaluated. This effectively restricted the registration to particular intensity ranges. Fourth, the multiresolution approach was examined for its ability to expedite the automated search algorithm. Fifth, the use of spatial masking was investigated to see whether it facilitates the registration of partially overlapping volumes. In all cases, registration experiments were performed with and without these modifications to determine their effect on the success of registration. Success was determined by comparing the results of these experiments to those of manual registration of the same images as described in the next section. Experiments with these parameters should provide insight into improving registration of MR and SPECT prostate images. We performed registration experiments using the SPECT and MRI image volumes from patients S4-S8.

\section{E. Registration Evaluations}

We evaluated registration experiments by visual inspection. We used RegViz, a program created in IDL (Interactive Data Language, Research System Inc., CO) in our laboratory with multiple visualization and analysis methods. First, we manually segmented prostate boundaries in image slices and copied them to corresponding slices. This enabled visual determination of the overlap of prostate boundaries over the entire volume. Second, color overlay displays were used to evaluate overlap of structures. One image was rendered in gray and the other in the "hot-iron" color scheme. To visualize potential differences, it was quite useful to interactively change the contribution of each image using the transparency scale. Third, we used a sector display, which divided the reference and registered images into rectangular sectors and created an output image by alternating sectors from the two input images. Even subtle shifts of edges would be clearly seen.

Our standard evaluation method for SV registration was to compare SV and VV registration. Since this relies on VV registration accuracy, we now review our previous results [7]. For volume pairs acquired over a short time span from a supine subject with legs flat on the table, prostates were well aligned and prostate centroid displacements were typically $<1 \mathrm{~mm}$. The registration accuracy as determined from displacements of pelvic bony landmarks was $1.6 \pm 0.2 \mathrm{~mm}$. From our success with
VV prostate registration, we decided that we could measure SV accuracy by comparing results to VV registrations for those volume pairs having low $\mathrm{VV}$ registration error. To compare $\mathrm{SV}$ and $\mathrm{VV}$ registration, we defined a rectangular volume of interest (VOI) just covering the prostate and calculated voxel displacements between the two registrations. To voxels within the VOI, we applied the transformations obtained by VV and by SV registrations. We then calculated the 3-D Euclidian displacements between the transformed voxels. The mean voxel distance was used as our metric of SV registration error. For the evaluation of algorithm robustness, we defined the SV registration as being successful when the 3-D displacement was less than $2.0 \mathrm{~mm}$.

The success of computer registration of SPECT and MRI volumes was determined by comparing results to manual registration. Manual registration was done by two board-certified nuclear medicine radiologists blinded to the automatic registration results. Manual registration was done using a software package with a graphical user interface (GUI) developed in-house, which allows graphical manipulation of volumes with six degrees of freedom in a rigid body registration. A color overlay was used to assess registration quality.

Two radiologists with a nuclear medicine specialty aligned the image volumes, and whenever there was a discrepancy, they reached a consensus for a single transformation. This painstaking cross-validation was a time-consuming process and certainly would not be a routine procedure, but the results served as the gold standard for the automated method. We defined a successful automatic registration to be obtained when all displacements were $<2$ voxels $(6 \mathrm{~mm})$ in the $\mathrm{x}, \mathrm{y}$, and $\mathrm{z}$ directions and angle differences were $<2$ degree for all angles about each of the three axes.

Although manual registration is difficult and somewhat operator dependent, it is the only acceptable option for an independent registration on the patient SPECT and MRI volumes. Skin fiducials would be of limited value in the pelvis, and there are no good identifiable point anatomical landmarks in the SPECT images.

We simulated the iMRI-guided procedures using our image registration and fusion software that are specially designed for this application. Before treatment, we acquired SPECT and high-resolution MRI volumes from the same patients. Second, we registered the two images and transferred the pair of aligned data sets to a workstation that was used for the slice to volume registration. Third, we connected the workstation to the iMRI scanner and obtained iMRI image slices from the scanner. Fourth, we performed the slice to volume registration. Finally, the software created fused images of the three modalities as would be done for image guidance. All registrations and image fusions are automatic.

\section{RESULTS}

\section{A. Registration of an iMRI Slice to a High-Resolution MRI Volume}

We determined SV registration results for slices near the prostate in the three standard orthogonal orientations. Comparing to VV, mean and standard deviation registration errors across 9 volume pairs were $0.4 \mathrm{~mm} \pm 0.2 \mathrm{~mm}$, 


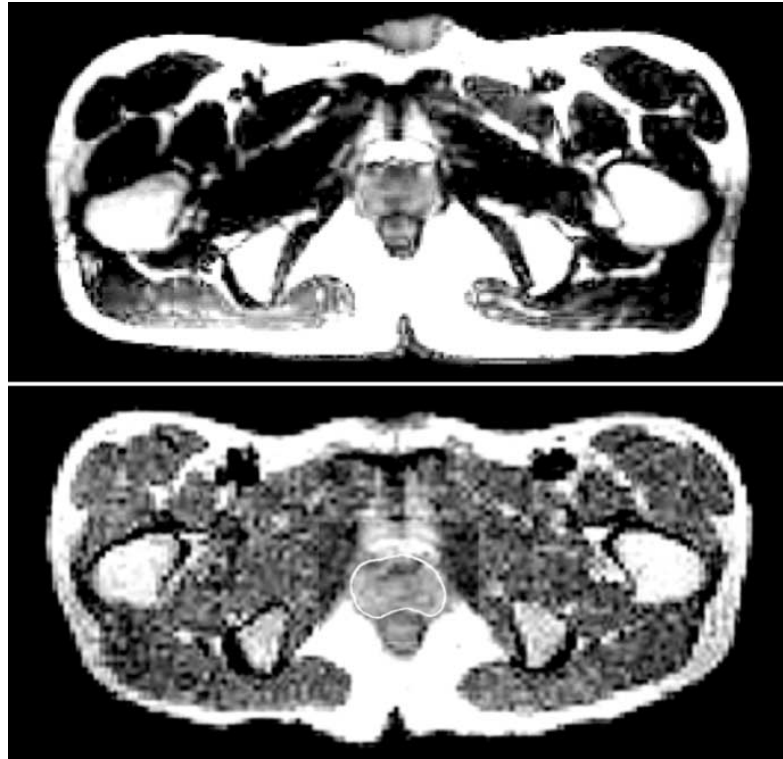

Fig. 1. Prostate MR images. The top is the high-resolution MR image. The bottom is the actual iMRI image; the rectangular window at the center is the transparency display of both images. The prostate boundary segmented from high-resolution MR image, matches well with the prostate in iMRI image. Images are from the volunteer S3.

$0.5 \mathrm{~mm} \pm 0.2 \mathrm{~mm}$, and $2.6 \mathrm{~mm} \pm 1.6 \mathrm{~mm}$ for transverse, coronal and sagittal slices covering the prostate, respectively. Transverse slices worked best because they contain many relatively rigid anatomical structures.

Simulation experiments showed SV registration to be very insensitive to noise. We performed over 100 registration experiments with noise added to give signal-to-noise ratio (SNR) ranging from 20 to 5 . Using the slice configurations recommended above (transverse slices near the prostate center), we obtained $100 \%$ successful registrations (an error $<2.0 \mathrm{~mm}$ ) for SNRs $\geq 10$, a value much worse than the clinical SNR value of $\approx 25$ on our iMRI system.

As for SV registration of actual iMRI image slices with a high-resolution MR volume, the contours overlap and overlay images show that the prostate matches very well (Fig. 1). Other visual inspection techniques also demonstrate excellent registration. Note that a single iMRI image was used to produce this registration result.

We now describe some aspects of the implementation. The time for an SV registration was typically about $3 \mathrm{sec}$ on a Pentium IV, $2.4 \mathrm{GHz}$ CPU, with 1 Gbytes of memory. The algorithm was written in IDL and could probably be made much faster in a lower level language such as $\mathrm{C}$.

\section{B. Registration of SPECT and High-Resolution MRI Volumes}

An example of a successful automatic registration is shown in Fig. 2. All anatomical features including the bone marrow in the femur and pubic symphysis are well aligned in the color overlay. This MR-SPECT volume pair and four others were successfully registered according to the criteria defined earlier. Standard algorithm parameters (Section II-C) were used with the lower-left quadrant of the joint histogram used for calculating MI. Successful image registration was obtained with images from patients S4, S5, and S8. There were four other MR-SPECT volume pairs obtained from patients S6 and S7 that were not successfully registered with our program. In all four cases, the MR images were not acquired using our final, optimized MR sequence (Section III-A). When we used the optimized sequence with full anatomical coverage, registration was always successful. We believe that automated SPECT-MRI registration will be feasible on many patients' images.

We now report the registration results of SPECT and high-resolution MRI images of the phantom. Registrations of the phantom images were carried out by displacing the aligned image pair with known rotation and translations. All orientations, axial, sagittal, and coronal, were successfully registered. Other experiments showed that intensity scaling and multiresolution could not improve the registration ability for both phantom and human data.

\section{Image Fusion and Visualization}

We created image registration and fusion software for the potential applications in iMRI-guided procedures. In Fig. 3, we demonstrate the image fusion visualization software in a simulation of clinical usage. SPECT and high-resolution MR images were acquired, transferred to a workstation, and registered prior to the "simulated" procedure. We then simulate acquiring thick iMRI slices, register them to the high-resolution volume, and prepare the visualization in Fig. 3. In this figure, one can see all. The registered images are shown in the three windows at the top line (Fig. 3). After registration, the program creates fused images as displayed at the bottom.

\section{DiscusSiON AND CONCLUSION}

This preliminary study has shown promising algorithmic results for bringing nuclear medicine, functional images into the interventional MRI suite. Automatic registration of SPECT and MRI volumes was always successful with "good" MRI volumes obtained using the optimized acquisition sequence and covering all anatomy of interest. Slice-to-volume automatic registration was even more successful with highly accurate, robust registration obtained. Putting these two steps together, a patient's SPECT images can be registered to a high-resolution MRI volume prior to an iMRI procedure; live-time iMRI slice images can be registered to the MRI volume; and, finally, one can then display the live-time iMRI slice image with the appropriately reformatted, fused image from the SPECT and high-resolution MRI image volumes.

The required registration accuracy is probably less than one might think. The live-time iMRI image obtained in the plane of the advancing needle will always be used for guiding a needle for intervention or biopsy. The corresponding fused SPECT-MRI and/or high-resolution MRI images will be used as a planning guide. With proper visualization tools, interventional radiologists should be able to mentally account for any small registration errors. In addition, there is often image evidence of cancer in MR prostate images that can perhaps be identified with the aid of functional images. Such MR-visible lesions can then become the markers for tumor targeting. Any potential gross registration errors should be easily recognized resulting in a failure to include the functional image data in the 


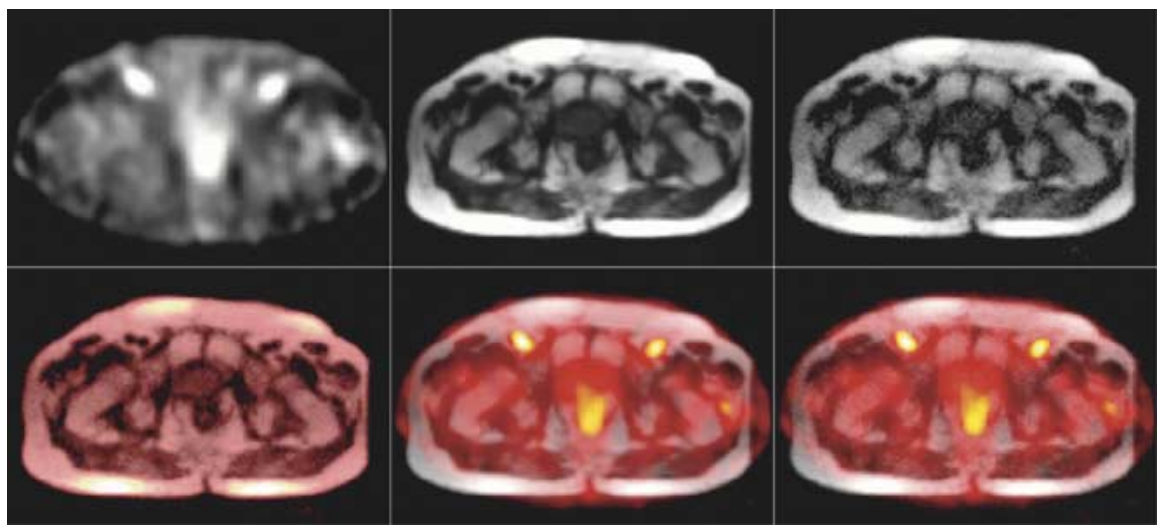

Fig. 2. Registration results of patient data. The top three images show corresponding registered SPECT, high-resolution MRI, and simulated iMRI images, respectively. The bottom three windows show the fused images of the three modalities, from left to right, iMRI/MRI, SPECT/MRI, and SPECT/iMRI, respectively. Images are from Patient S4.

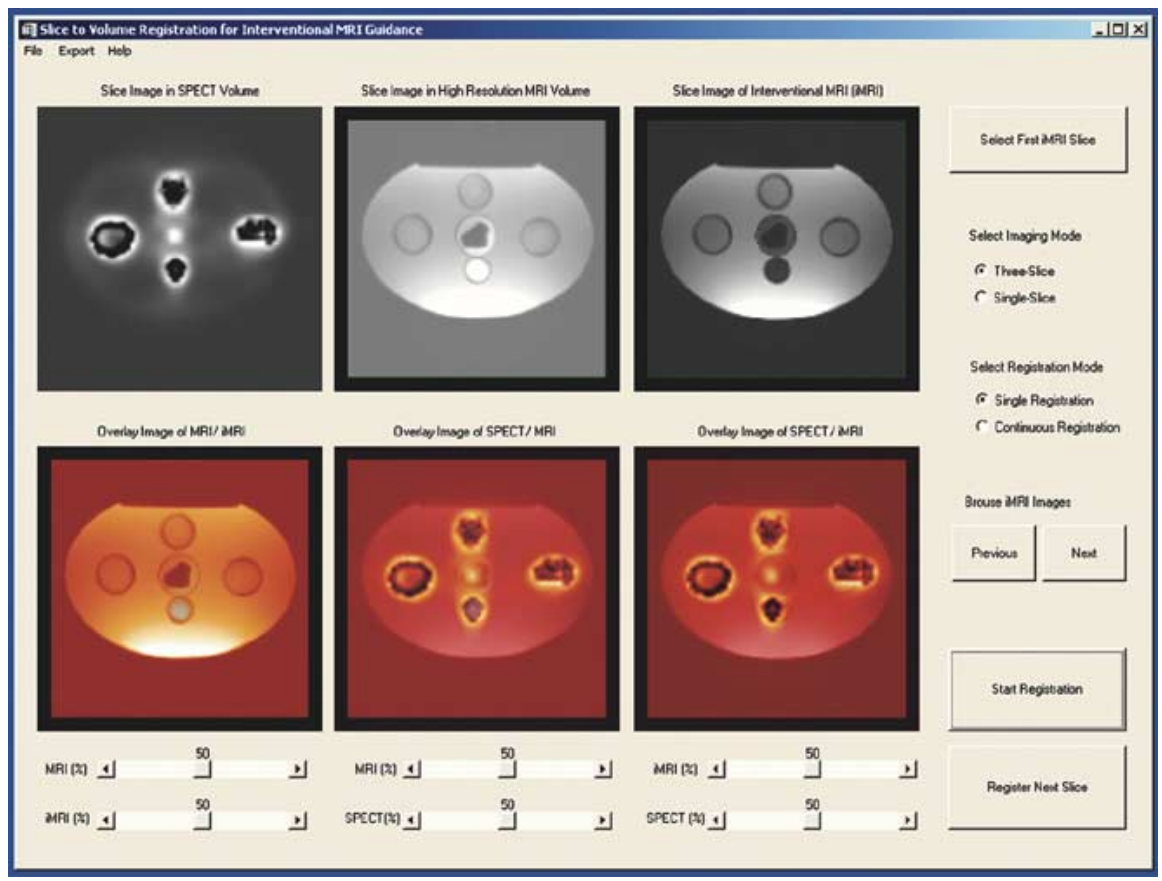

Fig. 3. Simulation experiments with phantom using registration and fusion software. The top three windows from left to right show corresponding registered SPECT, high-resolution MRI, and iMRI images, respectively. The bottom three windows from left to right show the fused images, iMRI/MRI, SPECT/MRI, and SPECT/iMRI, respectively. Other buttons and sliders control the configuration and registration.

iMRI suite but not in a catastrophic misguidance of the therapy needle.

More analysis of registration error is possible. The overall registration error of placing a SPECT image with a live-time iMRI image depends upon both SPECT-MRI and the slice-to-volume errors. The slice to volume error for voxels near the prostate is $\approx 1.4 \mathrm{~mm}$, as argued elsewhere [11], [12]. The SPECT-MRI error can be roughly estimated from the requirements for acceptable registration $\left( \pm 6 \mathrm{~mm}\right.$ and $\left.\pm 2^{\circ}\right)$. Slice-to-volume registration has an error less than obtained with SPECT-MRI, as would be expected from the low resolution and reduced number of features with SPECT. The automatic SPECT-MRI registration error is comparable to the uncertainty of manual registration. (After all, that is how we specified the requirements for "acceptability.") Despite such uncertainty, SPECT images have been routinely registered with CT and
MR images at our institution to use ProstaScint ${ }^{\circledR}$ for diagnostic studies of prostate cancer. If SPECT-MRI can be used for diagnostic procedures and slice-to-volume registration has much less error, then we anticipate that the combined process will fulfill needs for guidance as argued in the last paragraph.

To minimize registration error, we recommend that image data are obtained under comparable conditions by keeping a similar posture and by taking clinical measures to reduce rectal and bladder filling. Warping registration method may be useful to correct significant deformations at the expense of additional complexity, time, and possibly robustness [18], [19].

Finally, we believe that it is quite feasible to include previously acquired nuclear medicine SPECT images and high-resolution MRI data into iMRI-guided minimally invasive treatment procedures. We are beginning to explore this application in animal experiments. 


\section{REFERENCES}

[1] J. S. Lewin, C. F. Connell, J. L. Duerk, Y. C. Chung, M. E. Clampitt, J. Spisak, G. S. Gazelle, and J. R. Haaga, "Interactive MRI-guided radiofrequency interstitial thermal ablation of abdominal tumors: Clinical trial for evaluation of safety and feasibility," J. Magn. Resonance Imaging, vol. 8, pp. 40-47, 1998.

[2] D. L. Wilson, A. Carrillo, L. Zheng, A. Genc, J. L. Duerk, and J. S. Lewin, "Evaluation of 3D image registration as applied to MR-guided thermal treatment of liver cancer," J. Magn. Resonance Imaging, vol. 8, pp. 77-84, 1998.

[3] A. Carrillo, J. L. Duerk, J. S. Lewin, and D. L. Wilson, "Semiautomatic 3-D image registration as applied to interventional MRI liver cancer treatment," IEEE Trans. Med. Imaging, vol. 19, pp. 175-185, 2000.

[4] D. B. Sodee, N. Malguria, P. Faulhaber, M. I. Resnick, J. Albert, and G. Bakale, "Multicenter ProstaScint imaging findings in 2154 patients with prostate cancer," Urol., vol. 56, pp. 988-993, 2000.

[5] J. Scheidler, H. Hricak, D. B. Vigneron, K. K. Yu, D. L. Sokolov, L. R. Huang, C. J. Zaloudek, S. J. Nelson, P. R. Carroll, and J. Kurhanewicz, "Prostate cancer: Localization with three-dimensional proton MR spectroscopic imaging-Clinicopathologic study," Radiol., vol. 213, pp. 473-480, 1999 .

[6] R. K. Tenhaken, J. D. Forman, D. K. Heimburger, A. Gerhardsson, D. L Mcshan, C. Pereztamayo, S. L. Schoeppel, and A. S. Lichter, "Treatment planning issues related to prostate movement in response to differential filling of the rectum and bladder," Int. J. Radiat. Oncol. Biol. Phys., vol. 20, pp. 1317-1324, 1991.

[7] B. W. Fei, A. Wheaton, Z. Lee, J. L. Duerk, and D. L. Wilson, "Automatic MR volume registration and its evaluation for the pelvis and prostate," Phys. Med. Biol., vol. 47, pp. 823-838, 2002.

[8] H. Gray, Anatomy, Descriptive and Surgical (The Classic Collector's Edition). New York: Gramercy Books, 1977.

[9] A. Collignon, F. Maes, D. Delaere, D. Vandermeulen, P. Suetens, and G. Marchal, "Automated multimodality image registration using information theory," in Proc. 14th Int. Conf. (IPMI'95) Computational Imaging and Vision-Information Processing in Medical Imaging, Ile de Berder, France, 1995, pp. 287-298
[10] W. H. Press, S. A. Teukolsky, W. T. Vellerling, and B. P. Flannery, Numerical Recipes in C: The Art of Scientific Computing, 2nd ed. New York: Cambridge Univ. Press, 1992.

[11] B. W. Fei, J. L. Duerk, D. T. Boll, J. S. Lewin, and D. L. Wilson, "Slice to volume registration and its potential application tointerventional MRI guided radiofrequency thermal ablation of prostate cancer," IEEE Trans. Med. Imaging, vol. 22, pp. 515-525, 2003.

[12] B. W. Fei, J. L. Duerk, and D. L. Wilson, "Automatic 3D registration for interventional MRI-guided treatment of prostate cancer," Comput. Aided Surgery, vol. 7, pp. 257-267, 2002.

[13] F. Maes, A. Collignon, D. Vandermeulen, G. Marchal, and P. Suetens, "Multimodality image registration by maximization of mutual information," IEEE Trans. Med. Imaging, vol. 16, pp. 187-198, 1997.

[14] D. B. Sodee, R. J. Ellis, M. A. Samuels, J. P. Spirnak, W. F. Poole, C. Riester, D. M. Martanovic, R. Stonecipher, and E. M. Bellon, "Prostate cancer and prostate bed SPECT imaging with ProstaScint (R): Semiquantitative correlation with prostatic biopsy results," Prostate, vol. 37 , pp. $140-148,1998$

[15] A. Macovski, "Noise in MRI," Magn. Resonance Med., vol. 36, pp. 494-497, 1996.

[16] H. M. Hudson and R. S. Larkin, "Accelerated image-reconstruction using ordered subsets of projection data," IEEE Trans. Med. Imaging, vol. 13, pp. 601-609, 1994

[17] B. Likear and F. Pernus, "Registration of serial transverse sections of muscle fibers," Cytometry, vol. 37, pp. 93-106, 1999.

[18] B. W. Fei, C. Kemper, and D. L. Wilson, "Three-dimensional warping registration of the pelvis and prostate," in Proc. SPIE Medical Imaging on Image Processing, vol. 4684, San Diego, CA, 2002, pp. 528-537.

[19] _ - "A comparative study of warping and rigid body registration for the prostate and pelvic MR volumes," Computerized Med. Imaging Graphics, vol. 27, pp. 267-281, 2003. 
Copyright (C) 2004 IEEE. Reprinted from IEEE Transactions on Nuclear Science, Vol. 51, No. 1, February 2004

This material is posted here with permission of the IEEE. Internal or personal use of this material is permitted. However, permission to reprint/republish this material for advertising or promotional purposes or for creating new collective works for resale or redistribution must be obtained from the IEEE by writing to pubs-permissions@ieee.org. By choosing to view this document, you agree to all provisions of the copyright laws protecting it. 